\title{
Discovering manipulated social choices: The coincidence of cycles and manipulated outcomes
}

JOHN R. CHAMBERLIN

Institute of Public Policy Studies, 1516 Rackham Building, The University of Michigan, Ann Arbor, MI 48109

\section{Introduction}

In his recent book William Riker (1982) argues that the results of socialchoice theory require that we lower our traditional aspirations for the role of elections in democratic theory. In particular, he concludes that socialchoice theory sounds the death knell for the 'populist' interpretation of voting, the view that election outcomes embody the 'collective will' of the electorate. Other, more modest claims about elections also fall to the theorems of social-choice, until we remain with the extremely modest claim embodied in Riker's 'liberal' interpretation of voting. On this view elections provide the possibility of rejecting unpopular leaders, and the major value of elections is as a weapon against government tyranny.

Riker's argument proceeds in several stages. First, he reviews the analysis of social-choice in n-dimensional issue spaces. This literature conclusively demonstrates that the necessary and sufficient conditions for a majority voting equilibrium are so strict that one cannot expect them to ever be met. ${ }^{1}$ Thus Riker consigns the notion of a 'majority will' to the same 'dust bin' to which earlier liberals (from Madison to Bentley) had consigned Rousseau's general will.

Having discovered at the most abstract level that the search for a collective will of the electorate is pointless, for no such will exists, Riker proceeds to review the conclusions of social-choice theory about elections with a finite number of candidates. Here Arrow's theorem (1963) and its progeny take over. Not only do majority cycles still occur, but different systems with strong axiomatic credentials may select different candidates. Thus, even at this level the prospect is slight that we can endow election outcomes with great normative significance. But the prospects disappear almost entirely, in Riker's judgment, when we consider the manipulability theorems of social-choice theory. In his words: 
do not know whether this outcome truly reflects the values of the voters or whether it is the result of some kind of manipulation. (1978: 14)

... Since we cannot know whether manipulation has occurred, the truth and meaning of all outcomes is thereby rendered dubious ... Manipulated outcomes are meaningless because they are manipulated and unmanipulated outcomes are meaningless because they cannot be distinguished from manipulated ones. (1982: 236-237)

The destruction of the populist ideal is now complete. Its very coherence disintegrates at the abstract level, and even the more modest claim, that election outcomes are reflections of a 'majority will' on a given set of candidates, is defeated because we cannot remove the shadow of doubt that the manipulability theorems cast over all election outcomes. In the face of this evidence, Riker retreats to the liberal interpretation of voting: elections are important in a democratic society because they offer a possibility of rejecting unpopular leadership.

This essay tries to recapture at least some middle ground from Riker's claim by arguing that the 'ineradicable uncertainty' (1978: 15) that follows from the possibility of manipulation is more eradicable than Riker leads us to believe. Such an argument cannot by itself reestablish earlier claims for elections since it addresses only the final stage of Riker's argument. But if we have reason to believe that the other stages of Riker's argument are also rebuttable, as I suggest by citing the work of others, then a counter to the manipulation argument, in combination with those other counterarguments, will do much to restore the claim that we can interpret voting outcomes as meaningful expressions of the will of the majority of voters concerning a given set of candidates. If this argument fails to revive fully the populist vision, as it must, nevertheless it claims a deeper role for elections in democratic theory than does Riker's liberal alternative.

\section{Stability in majority voting}

The theorems that demonstrate the hopeless prospects for the stability of majority rule (Plott, 1967; McKelvey, 1976; Schofield, 1978) deal by far the greatest damage to the populist ideal. These theorems establish the implausibility of there being an abstract majority will that an election might reveal. But in an important recent paper, Nicholas Miller (1983) argues convincingly that the inherent intransitivity of majority choice, which seems so pathological from the standpoint of social-choice theory, is the very characteristic that leads many political theorists to see in a pluralist political system the promise of a stable democratic society. Briefly, Miller argues that pluralism rests its claim for long-run stability on the observation that losers in any given election have reason to believe that hard work and clever 
strategy between elections can yield a real chance of their being winners in the next election. This 'wait til' next year' spirit could not thrive, Miller asserts, in a society in which a stable majority will prevails, in the sense that social-choice theory seeks. Miller thus sees the short-term instability that plagues social-choice theory to be the very characteristic of a pluralist society that insures its long-run political stability. Miller's claim is thus not that the theorems are wrong, or that Riker's inferences from them are mistaken, but that when viewed from another vantage point, we might not choose to have it any other way. Miller's insightful analysis dispels some of the sense of doom that social-choice literature casts over elections in Riker's interpretation.

Having ascertained that the absence of majority equilibrium is both inevitable and perhaps functional after all, what can we say for elections at the next level? Here, the democratic process includes periodic elections among given (finite) sets of candidates. If majority winners exist for a series of such elections, and a voting system can reliably select them, then the claim that these voting outcomes are politically legitimate is a strong one. Several barriers to this view of elections remain, but none seems insurmountable. Voting cycles may still exist, but their frequency may be sufficiently low to make them unimportant. Many voting systems can select majority winners if they exist, and these systems are susceptible to manipulation via alterations in the set of candidates only if a voting cycle can be manufactured, a task that is not straightforward in real elections. Hence, we arrive at the problem of manipulation, which Riker believes is decisive. This essay tries to restore hope for a more fully democratic interpretation of election outcomes by showing, for at least one voting system, that we can substantially eradicate the uncertainty that the possibility of manipulation creates for the legitimacy of elections outcomes. If this is the case, then it is reasonable to view election outcomes as expressions of a majority will among the given candidates.

The analysis focuses on the performance of three voting systems in elections involving 1,000 voters and four candidates. ${ }^{2}$ Of greatest interest is the Kemeny system, which selects a winning ordering of the candidates. It proceeds by counting the number of 'pairwise switches' among candidates that would be necessary to convert all voter preferences into a single preference ordering. The ordering that would require the fewest switches is the winning ordering. Kemeny (1959) shows that the number of switches meets the axioms for a distance metric, and that the winning Kemeny ordering is in this sense the ordering that is 'closest' to the given set of voter preferences. Levenglick (1975) shows that the Kemeny system has an impressive set of axiomatic credentials, including symmetry, faithfulness, consistency, and the Condorcet criterion. In light of Levenglick's analysis, the Kemeny system meets our standards of how a voting system ought to behave. This 
study compares the Kemeny system with plurality voting and the Borda rule to compare its performance with those of the system we now use most often and a system that also has impressive (but somewhat different) axiomatic credentials (Young, 1974). My results indicate that Kemeny succeeds where the others fail, and they strongly suggest that the Kemeny method is capable of solving the problems of social-choice theory as well as any other system, and that we can place greater normative emphasis on election outcomes than Riker's liberal interpretation would allow.

I will refer to the winner of an election in which voters express their true (or 'sincere') preferences as the 'sincere' winner. An election is 'manipulated' when preference misrepresentation by voters leads to the selection of a candidate other than the 'sincere' winner. The key to our analysis is the relationship between the twin demons of social-choice theory - voting cycles and manipulated outcomes - a relationship that socialchoice theorists seem not to have noticed. Under certain circumstances these events are more likely to occur together than alone. This observation lies at the heart of the Bayesian analysis constructed here, which shows that whether or not a majority-voting cycle occurs is, under a wide variety of circumstances, an accurate indication of whether or not the outcome of the election has been manipulated. This analysis suggests that Riker's claim, that the 'truth and meaning of all outcomes is thereby rendered dubious,' (1982: 236) is too sweeping, for it will often be possible to eliminate much of the uncertainty concerning the 'sincerity' of the outcome by observing whether or not a cycle has occurred.

To a great extent, the strength of Riker's conclusion rests on the uncertainty that surrounds the question of whether voters have expressed their sincere (true) preferences. For Riker, this uncertainty is ineradicable, because 'to prove that strategic voting occurs, one must know what voters thought as well as what they did. But observers can see only the deed, never the thought' (1978: 15). But this is not the only way of obtaining information about the sincerity of voters' deeds. If we can observe something other than the outcome of voting, and if this piece of information is a reliable signal about the sincerity of the outcome (i.e., whether the outcome differs from that which would obtain if all voters cast ballots containing sincere preferences), then the uncertainty about the legitimacy of the outcome diminishes considerably.

The argument and the examples to follow will demonstrate that it is often possible to decrease greatly the uncertainty concerning whether or not the winner of an election is indeed the 'sincere' winner by observing a very simple signal: whether or not an intransitive majority voting cycle has occurred. ${ }^{3}$ Most studies of the likelihood of voting cycles among sincere preferences suggest that it is reasonably small, and work on spatial models shows the likelihood to be very small indeed. ${ }^{4}$ On the other hand, the 
strategies that manipulate election outcomes often create cycles in the process. The occurrence of a majority voting cycle in an actual election can thus be an imperfect signal of two things - a cycle in the sincere preferences or a manipulated outcome (or both). My analysis, which uses estimates of various probabilities derived from Monte Carlo methods, strongly suggests that in some kinds of electoral environments, and if certain kinds of socialchoice functions are in use, whether or not a voting cycle exists can be a decisive signal concerning the likelihood that the election has selected the 'sincere' winner.

It is possible for the three voting methods to select different winners, given the same set of preferences. Since the question of which candidate ought to win is irresolvable in light of Arrow's theorem and others, I investigate below only the probability of selecting the 'sincere' winner, that candidate who would be selected by a particular voting method given a sincere expression of preferences. It is worth noting that all three socialchoice methods share one property that makes them particularly worthy of study: it is quite easy in each case to articulate a strategy for manipulating a voting outcome that is easily understood and is often an optimal strategy.

\section{The model}

Manipulating an election outcome ordinarily is a very complicated matter. One must estimate the voters' preferences and one must usually estimate their actions as well, for they may not vote sincerely. One way to study the question is to adapt the line of work Farquharson (1969) began on voting on candidates under a fixed agenda. One could seek to identify sophisticated voting equilibria for the election games under the different voting systems, and to evaluate the properties of these equilibria. Such an analysis assumes perfect information about voter preferences and assumes that each voter carries out the game-theoretic analysis for herself. In light of what we know about the actions of voters in mass elections, both of these assumptions seem heroic, and the results of such an analysis may bear little resemblance to the outcomes of naturally occurring elections in which preferences are misrepresented. I develop instead a model that recognizes that less than perfect information will be available and that the average voter is unlikely to have either the ability or the incentive to carry out the complex analysis necessary to find equilibrium strategies. This model assumes that a candidate's campaign staff develops a manipulation strategy and then communicates it to the candidate's supporters. To flesh out the model, imagine that a candidate's campaign staff has access to reliable information about who the likely sincere winner (candidate A) and runner-up (candidate B) will be, but no other information about the structure of voters' preferences. ${ }^{5} \mathrm{My}$ 
analysis focuses on attempts to manipulate B into the winners's spot. Candidate B's staff must devise a manipulation strategy and communicate it to those voters who might join in the attempted manipulation on the basis of only this information. In mass elections the number of voters will be large and voters are likely to be geographically dispersed. These characteristics necessitate a general strategy that all who join in the attempted manipulation can follow, rather than a complex, multi-faceted strategy of the kind that might be possible with face-to-face communication among a few voters. Under these circumstances it seems very likely that candidate B's staff will communicate with voters thus: ${ }^{6}$

Plurality: $\quad$ 'If you prefer B to A, do not waste your vote on an also-ran, vote for B.'

Borda/Kemeny: 'If you prefer B to A, cast a ballot with B at the top and A at the bottom. This is your best hope of avoiding the election of A.'

The mere attempt to communicate these strategies does not guarantee their implementation. Some voters may be inattentive and never receive the message, and others may be lackadaisical or suspicious and not follow the strategy. For these reasons I treat the degree of compliance with the strategies as a variable. The model also includes the possibility that A's supporters may attempt to 'counter-manipulate' by casting ballots that reverse the positions of $A$ and $B$ in the strategies just given. Incorporating this response by A's supporters into the model provides some of the symmetric flavor of the equilibrium approach, but it does so in a manner that does not make unrealistic assumptions about voters' actions. Since the degrees of compliance with these strategies are variables, the range of possible actions that can result is obviously infinite, but for convenience I restrict the examples to the consideration of only eight possible 'states of nature,' each of which is characterized by the proportion of ' $B$ 's supporters' and 'A's supporters' that follow their respective strategies as just outlined. Table 1 gives the states to be considered in the examples. The analysis is generalizable to as many states as seems necessary in any instance. State 1 is the case of sincere voting. States 4,6 , and 8 indicate a degree of balance in the misrepresentation of preferences. A prior subjective probability distribution defined over these states is a basic input to the analysis. The following notation will be useful in developing the model:

W: the actual winner (for a given voting system);

$\mathrm{SW}$ : the sincere winner (for the same voting system);

C: the existence of a cycle in the expressed preferences of the voters ( $\overline{\mathrm{C}}:$ no cycle in the expressed preferences);

SC: the existence of a cycle in the sincere preferences of the voters. 
Table 1. States of nature - Proportion of supporters who cast ballot in accordance with manipulation/counter-manipulation strategies

\begin{tabular}{lll}
\hline State & B's supporters & A's supporters \\
\hline 1 & 0 & 0 \\
2 & 1 & 0 \\
3 & $1 / 2$ & 0 \\
4 & $1 / 2$ & $1 / 2$ \\
5 & $1 / 4$ & 0 \\
6 & $1 / 4$ & $1 / 4$ \\
7 & $1 / 10$ & 0 \\
8 & $1 / 10$ & $1 / 10$ \\
\hline
\end{tabular}

We observe the outcome of an election (either $(W, C)$ or $(W, \bar{C})$ ), and we would like to know these probabilities: ${ }^{7}$

$$
\begin{array}{ll}
\mathrm{P}(\mathrm{W}=\mathrm{SW} \mid \mathrm{C}) & \mathrm{P}(\mathrm{W}=\mathrm{SW} \mid \overline{\mathrm{C}}) \\
\mathrm{P}(\mathrm{SC} \mid \mathrm{C}) & \mathrm{P}(\mathrm{SC} \mid \overline{\mathrm{C}}) .
\end{array}
$$

As the examples to be presented show, whether or not a cycle exists sometimes will be sufficient to clear up much of the uncertainty that Riker considers to be ineradicable. The probabilities we wish to determine are posterior probabilities, and they may be calculated using Bayes's rule if the associated likelihood functions are known and if a prior distribution over the states of voting decisions is provided. Let $P(S=J)$ denote this prior distribution.

We can calculate the first posterior probability listed (the probability that the outcome has been manipulated given that a cycle exists) in either of the two ways:

$$
\mathrm{P}(\mathrm{W} \neq \mathrm{SW} \mid \mathrm{C})=\frac{\left.\sum_{\mathrm{J}(\mathrm{C} \mid \mathrm{W}} \neq \mathrm{SW}, \mathrm{S}=\mathrm{J}\right) \mathrm{P}(\mathrm{W} \neq \mathrm{SW} \mid \mathrm{S}=\mathrm{J})}{\underset{\mathrm{J}}{\mathrm{P}(\mathrm{C} \mid \mathrm{S}=\mathrm{J}) \mathrm{P}(\mathrm{S}=\mathrm{J})}}
$$

and

$$
\mathrm{P}(\mathrm{W} \neq \mathrm{SW} \mid \mathrm{C})=\sum_{\mathrm{J}} \mathrm{P}(\mathrm{W} \neq \mathrm{SW} \mid \mathrm{C}, \mathrm{S}=\mathrm{J}) \mathrm{P}(\mathrm{S}=\mathrm{J} \mid \mathrm{C}),
$$

in which $P(S=J / C)$ is the posterior probability of state $J$ given that a cycle has occurred. It is given by

$$
\frac{P(C \mid S=J) P(S=J)}{\sum_{J} P(C \mid S=J) P(S=J)} \text {. }
$$


The likelihood functions in the numerator must be estimated, in my case on the basis of Monte Carlo methods, and we assess the prior distribution directly based on what we know or suspect about the election in question. We can derive similar expressions for the other probabilities.

\section{Results}

The following examples give posterior probabilities for the three voting systems under varying prior distributions listed in Table 2 . These represent a dozen interesting sets of plausible conditions. ${ }^{8}$ The first four cases contain a range of values in which no counter-manipulation occurs. The remaining eight cases allow for varying degrees of misrepresentation and countermisrepresentation. Among the interesting comparisons are B-H, C-E, and D-G, which contrast equivalent situations with and without counter-misrepresentation.

As Chamberlin and Cohen (1978) and Merrill (1984) demonstrate, variation in the structure of collective preferences can greatly affect the performance of social-choice functions. To check the robustness of the conclusions that follow to variation in the environment, I study three environments. In each case, I use 900 elections (each with four candidates and 1000 voters) to estimate the necessary likelihood functions. The first environment is an impartial culture, in which all 24 possible preference orders are equally likely to occur. As the second environment I use the spatial elections Chamberlin and Cohen (1978) generate. These elections come from a four-dimensional spatial model with a specified covariance structure among the dimensions, and they use the Euclidean distance between candidate and voter positions to establish preference orderings. ${ }^{9}$ The third environment

Table 2. Prior probability distributions

\begin{tabular}{lllllllll}
\hline \multicolumn{2}{l}{ States of nature } & & & & & \\
\hline Prior & 1 & 2 & 3 & 4 & 5 & 6 & 7 & 8 \\
\hline A & .2 & .2 & .2 & & .2 & & .2 & \\
B & .6 & & & & .2 & & .2 & \\
C & .8 & & & & & & .2 & \\
D & .1 & & & & & & .9 & \\
E & .8 & & & & & & .1 & .1 \\
F & .4 & & & & & & .3 & .3 \\
G & .1 & & & & & & .45 & .45 \\
H & .6 & & & & .1 & .1 & .1 & .1 \\
I & .2 & & & & .2 & .2 & .2 & .2 \\
J & .4 & & & & .2 & .1 & .2 & .1 \\
K & .4 & & .1 & .1 & .1 & .1 & .1 & .1 \\
L & .2 & & .2 & .2 & .2 & .2 & & \\
\hline
\end{tabular}


consists of elections that are a ' $50-50$ ' mixture of voters from an impartialculture model and voters from the Chamberlin-Cohen spatial model. ${ }^{10}$

Tables 3 and 4 show the effects on voting outcomes under the conditions encoded in the prior distributions of Table 2 (Results appear to three decimal places only if this is necessary to avoid rounding off to zero). The first result worthy of note is the differences across the three electoral environments. Both cycles and manipulated outcomes occur more frequently in the impartial-culture elections than in the spatial elections. The ' $50-50$ ' elections are the intermediate case, as expected. These results confirm the importance of assumptions about the structure of collective preferences to empirical conclusions about social-choice.

The tables contain the 'base-rate' figures that give rise to Riker's concerns. In the absence of any further indication of whether or not misrepresentation has occurred, these tables suggest the frequencies with which we could expect to observe cycles and manipulated outcomes under the various conditions. With respect to manipulated outcomes, the Kemeny function is generally the most successful in selecting sincere winners, with plurality not far behind. These frequencies seem large enough to warrant Riker's level of concern, since the possibility of manipulated outcomes is real under all conditions, and under the impartial-culture assumption it is quite large. The frequencies of cycles contribute to the problem. Because of the manipulation strategy involved, plurality escapes most of the difficulty on this count, and since rank-ordered preferences would never be collected in the first place, its performance on this criterion is probably best ignored. But the Borda and Kemeny systems have substantial frequencies of cycles in all but the spatial case. The occurrence of cycles with such frequencies would certainly threaten the legitimacy of election outcomes, since with both of these methods the occurrence of a cycle will not slip by unnoticed because the data will be available to check for cycles.

These tables add support for the potential chaos, whether observed or not, that Riker sees as inherent in the populist view of democratic choice. If these results were our only way of assessing the situation, Riker's pessimistic conclusions would be difficult to reject. But the possibility of using the occurrence of a cycle as a signal about the occurrence of a manipulated outcome allows us to resolve substantially the uncertainty that clouds the legitimacy of the outcomes of specific elections (at least in the case of the Kemeny function). Tables $5 \mathrm{a}, 5 \mathrm{~b}$, and $5 \mathrm{c}$ show the posterior probabilities of a manipulated outcome and/or a sincere cycle conditional upon the observance or non-observance of a cycle. In general, the greater the probability of misrepresentation of preferences, and the greater the degree of misrepresentation, the greater is the likelihood of a cycle and/or a manipulated outcome. The tables also indicate that using $C$ or $\bar{C}$ as a signal has varied results. In the case of plurality voting, it is a relatively weak signal 
Table 3. Frequencies of manipulated outcomes

\begin{tabular}{|c|c|c|c|c|c|c|c|c|c|}
\hline \multirow[t]{2}{*}{ Prior } & \multicolumn{3}{|c|}{$\begin{array}{l}\text { Impartial culture } \\
\mathrm{P}(\mathrm{W} \neq \mathrm{SW})\end{array}$} & \multicolumn{3}{|c|}{$\begin{array}{c}\text { Spatial } \\
\mathrm{P}(\mathrm{W} \neq \mathrm{SW})\end{array}$} & \multicolumn{3}{|c|}{$\begin{array}{l}50-50 \\
P(W \neq S W)\end{array}$} \\
\hline & $\mathbf{P}$ & B & $\mathbf{K}$ & $\mathbf{P}$ & B & $\mathbf{K}$ & $P$ & $\mathbf{B}$ & $\mathbf{K}$ \\
\hline Sincere & .00 & .00 & .00 & .00 & .00 & .00 & .00 & .00 & .00 \\
\hline A & .76 & .61 & .67 & .26 & .50 & .29 & .47 & .61 & .40 \\
\hline B & .36 & .37 & .27 & .05 & .18 & .05 & .13 & .23 & .09 \\
\hline $\mathrm{C}$ & .16 & .17 & .08 & .01 & .06 & .01 & .03 & .09 & .02 \\
\hline $\mathrm{D}$ & .72 & .76 & .35 & .05 & .26 & .05 & .15 & .41 & .08 \\
\hline $\mathrm{E}$ & .08 & .09 & .04 & .01 & .03 & .01 & .02 & .05 & .01 \\
\hline $\mathbf{F}$ & .26 & .24 & .12 & .03 & .09 & .02 & .06 & .14 & .03 \\
\hline G & .38 & .39 & .18 & .04 & .14 & .02 & .09 & .21 & .04 \\
\hline $\mathbf{H}$ & .20 & .19 & .14 & .04 & .09 & .03 & .08 & .12 & .05 \\
\hline I & .41 & .39 & .27 & .07 & .19 & .05 & .16 & .24 & .09 \\
\hline $\mathrm{J}$ & .38 & .38 & .27 & .06 & .18 & .05 & .14 & .24 & .09 \\
\hline $\mathbf{K}$ & .31 & .30 & .24 & .09 & .17 & .07 & .18 & .22 & .11 \\
\hline $\mathbf{L}$ & .46 & .43 & .39 & .17 & .29 & .13 & .31 & .35 & .20 \\
\hline
\end{tabular}

Table 4. Frequencies of cycles

\begin{tabular}{|c|c|c|c|c|c|c|c|c|c|}
\hline \multirow[t]{2}{*}{ Prior } & \multicolumn{3}{|c|}{$\begin{array}{c}\text { Impartial culture } \\
P(C)\end{array}$} & \multicolumn{3}{|c|}{$\begin{array}{l}\text { Spatial } \\
P(C)\end{array}$} & \multicolumn{3}{|c|}{$\begin{array}{l}50-50 \\
P(C)\end{array}$} \\
\hline & $\mathrm{P}$ & B & K & $\mathbf{P}$ & B & $\mathbf{K}$ & $\mathbf{P}$ & B & K \\
\hline Sincere & .15 & .15 & .15 & .004 & .004 & .004 & .01 & .01 & .01 \\
\hline $\mathbf{A}$ & .12 & .62 & .75 & .02 & .30 & .36 & .09 & .42 & .49 \\
\hline B & .13 & .35 & .42 & .01 & .06 & .08 & .02 & .12 & .14 \\
\hline $\mathrm{C}$ & .14 & .22 & .26 & .004 & .02 & .02 & .02 & .04 & .05 \\
\hline D & .11 & .48 & .65 & .01 & .06 & .08 & .03 & .12 & .16 \\
\hline $\mathrm{E}$ & .13 & .18 & .20 & .004 & .01 & .01 & .02 & .03 & .03 \\
\hline $\mathrm{F}$ & .09 & .25 & .30 & .004 & .02 & .03 & .02 & .05 & .06 \\
\hline$G$ & .07 & .30 & .38 & .004 & .03 & .04 & .03 & .07 & .09 \\
\hline $\mathrm{H}$ & .11 & .24 & .27 & .01 & .03 & .04 & .02 & .07 & .08 \\
\hline I & .08 & .34 & .40 & .01 & .06 & .08 & .03 & .12 & .14 \\
\hline $\mathbf{J}$ & .11 & .34 & .41 & .01 & .06 & .08 & .03 & .12 & .14 \\
\hline $\mathbf{K}$ & .09 & .30 & .34 & .01 & .08 & .10 & .03 & .13 & .15 \\
\hline $\mathbf{L}$ & .08 & .38 & .43 & .08 & .14 & .17 & .06 & .22 & .26 \\
\hline
\end{tabular}

concerning manipulated outcomes but a relatively strong signal concerning the existence of a sincere cycle. For the Borda and Kemeny systems, the results are reversed. Only in the case of the Kemeny function, however, is the signal extremely decisive. For the Kemeny system, if a cycle is not observed, one can be reasonably certain that the sincere winner has been 


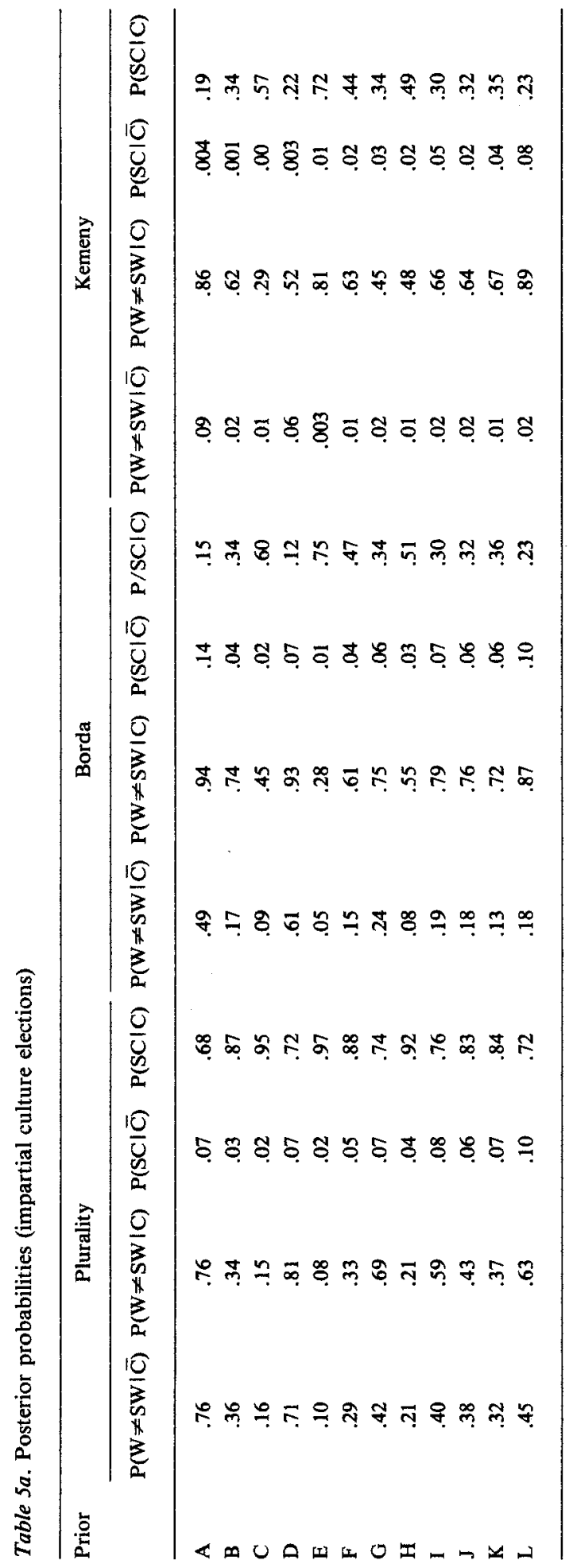




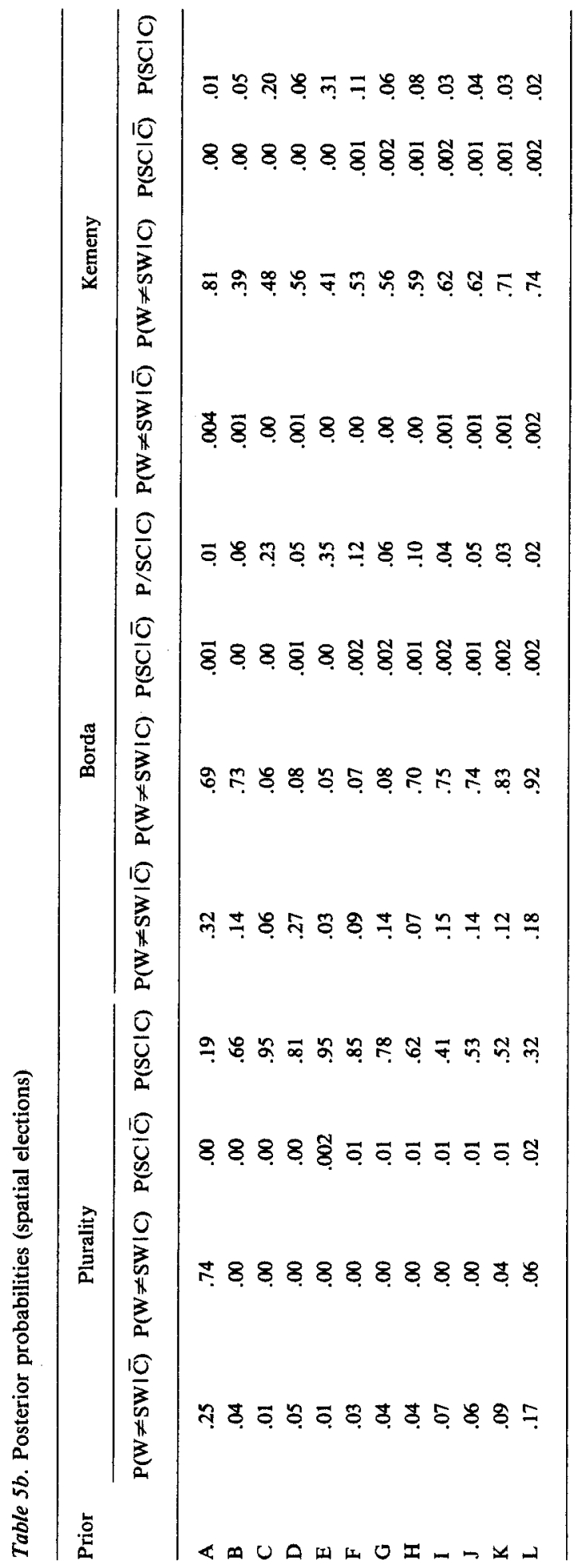




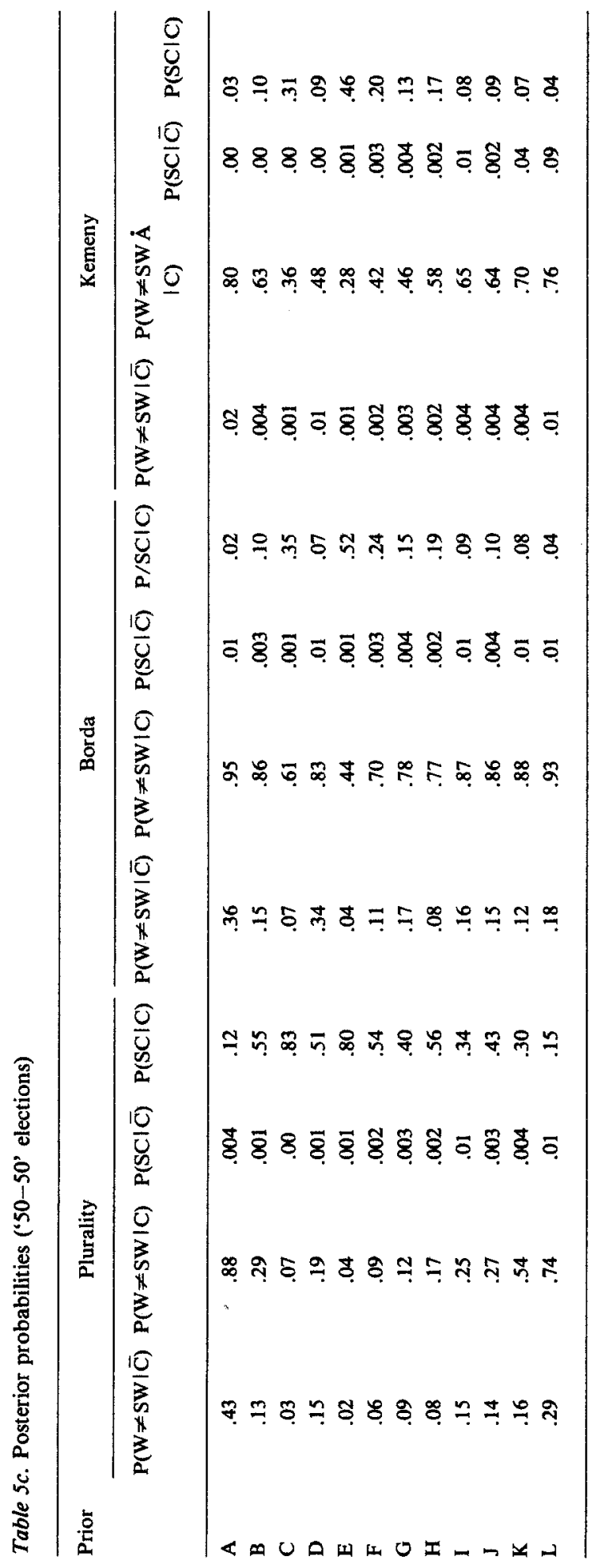


chosen and that this winner is a sincere Condorcet winner. Since cycles are reasonably unlikely to occur, we can accept the vast majority of Kemeny outcomes for what we wish them to be - the sincere indication of the wishes of the majority of voters. In these instances the uncertainty that gives rise to Riker's pessimism seems to be genuinely eradicated.

The uncertainty is resolved less if we observe a cycle. For plurality voting, a cycle tells us very little about the occurrence of a manipulated outcome, but it is a fairly reliable signal of a sincere cycle. This result occurs because the manipulation strategy for the plurality system, when successfully implemented, is much less likely to generate cycles. Except for the spatial elections, a cycle with the Borda rule is a reasonably strong signal of a manipulated outcome. For the Kemeny system the signal is weaker than it is for the Borda system, except for the spatial elections. In no case does the strength of the signal approach that for the Kemeny function in the absence of a cycle.

It is possible to compute several measures that indicate the overall strength of using $\mathrm{C}$ or $\overline{\mathrm{C}}$ as a signal of manipulated outcomes. For instance, one can compute the proportion of elections in which a rule using the signal to predict manipulated outcomes does so correctly. Such a rule would be:

1. if a cycle has occurred, and $P(W \neq S W \mid C)>.5$, guess that the outcome was manipulated;

2. if a cycle has not occurred, and $P(W \neq S W \mid \bar{C})>.5$, guess that the outcome was manipulated

3. otherwise, guess that the outcome is sincere.

Using this rule, the probability of a correct guess is:

$$
\begin{aligned}
\mathrm{P}^{*}= & \max [\mathrm{P}(\mathrm{W} \neq \mathrm{SW} \mid \mathrm{C}), 1-\mathrm{P}(\mathrm{W} \neq \mathrm{SW} \mid \mathrm{C})] * \mathrm{P}(\mathrm{C})+ \\
& \max [\mathrm{P}(\mathrm{W} \neq \mathrm{SW} \mid \overline{\mathrm{C}}), 1-\mathrm{P}(\mathrm{W} \neq \mathrm{SW} \mid \overline{\mathrm{C}})] * \mathrm{P}(\overline{\mathrm{C}}) .
\end{aligned}
$$

The Goodman-Kruskal lambda $(\lambda)$ gives another measure of the signal's strength, which indicates the reduction in the error rate that results from using a signal. This reduction is given by $\lambda=[\mathbf{P}-(1-\mathrm{P} *)] / \hat{\mathrm{P}}$, in which $\hat{\mathbf{P}}$ $=\min [\mathrm{P}(\mathrm{W} \neq \mathrm{SW}), 1-\mathrm{P}(\mathrm{W} \neq \mathrm{SW})]$ is the error rate if one were to guess on each occurrence the outcome most likely to occur. Table 6 shows the values of these two measures. Notice that in all but one case (prior D for the impartial culture elections), the Kemeny system has the largest value of $P$ (correct). Notice also that $\lambda$ is non-zero $19 \%, 75 \%$, and $67 \%$ of the time for the plurality, Borda, and Kemeny systems, respectively. For the last two systems, the signal very significantly improves the prediction rate, because the manipulation strategy, if successful, generally results in a voting cycle. It must do so in the case of the Kemeny system, unless one already exists. This observation explains why $\mathrm{C}$ is a stronger signal of a sincere outcome for the Kemeny system than the Borda system, it also explains why, when 


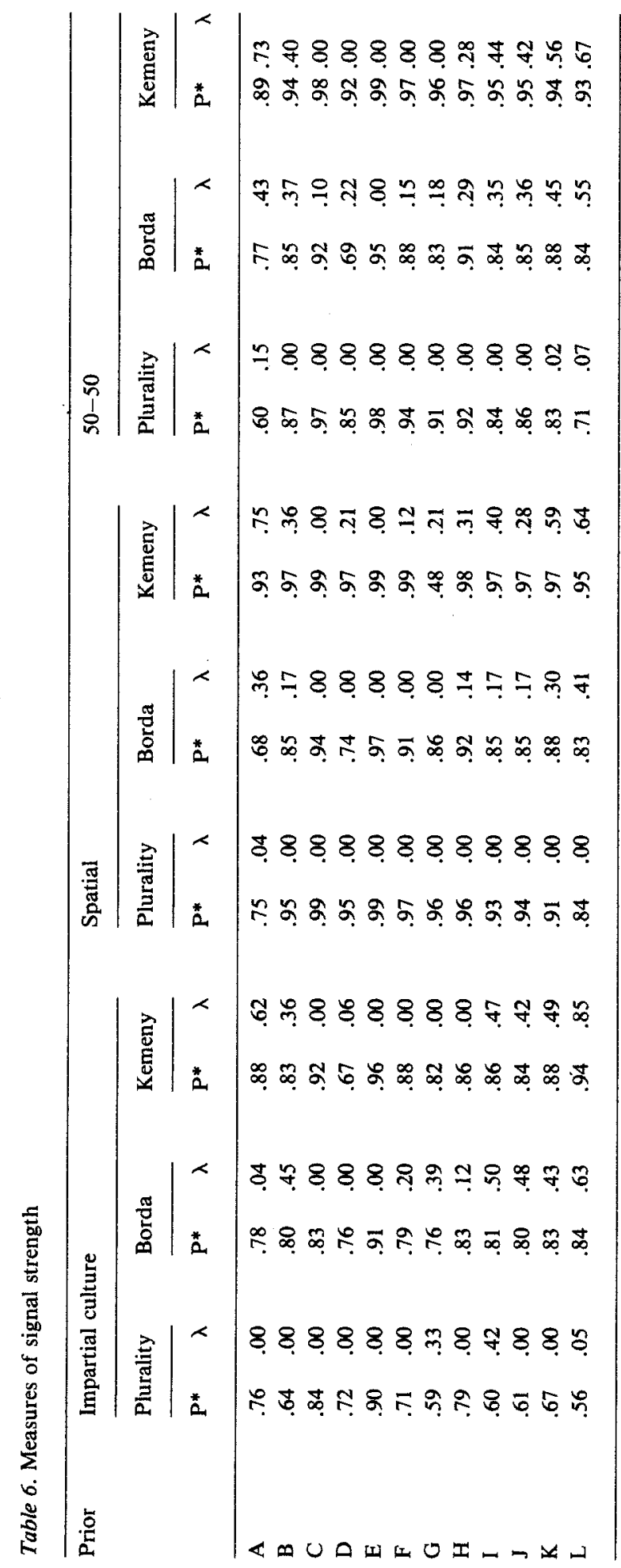


$\lambda$ is non-zero for the Kemeny system, it is almost always greater than the value of $\lambda$ for the Borda rule.

\section{Discussion}

Riker's pessimism about the claims that one can make about voting outcomes springs from several sources: the absence of equilibria in multidimensional representations of the problem; the lack of any convincingly 'best' voting system; and the omnipresent threat of manipulability. Faced with these problems, Riker rejects what he calls the populist view of voting outcomes as the expression of a collective will, in favor of what he calls the liberal interpretation of voting, which simply requires that voting processes have some chance of throwing rascals out of office. Given the heavy normative price that one pays in adopting such a conclusion, it seems to me that it should be done reluctantly and only in the face of convincing evidence. For Riker, the 'ineradicable uncertainty' surrounding the sincerity of voting outcomes is a crucial piece of evidence. The results presented here strongly suggest that Riker's judgment about the threat of manipulation is too sweeping, and that at least in the case of the Kemeny voting system, the problem can be overcome.

The claim that taking a Bayesian view of this problem allows one to erase the uncertainty concerning manipulation may strike some readers as missing Riker's point, for part of his argument, surely, is that we could never reliably assess the prior probability distribution necessary for the calculations. To do so would require us to do the very thing that he suggests we cannot do: assess the thought as well as the deed. This claim seems true, but the claim for the Kemeny function is that its ability to signal sincere outcomes is robust over all of the priors I investigate. If this property is general, then doubts about our ability to assess reliably the prior distribution are not telling ones.

The key to the Kemeny function's success is that it always selects a Condorcet winner if one exists. Manipulation of this system requires, therefore, that a cycle be established if one does not already exist. A manipulated outcome can occur in the absence of a cycle only if a cycle exists in the sincere preferences and if it was undone in the process of manipulation. This observation, combined with the observation that sincere cycles are relatively rare, is what makes the absence of a cycle a nearly flawless signal that the electorate chose the sincere winner. ${ }^{11}$

Under most of the conditions studied, a cycle usually will not occur, and in these cases we can be nearly certain that the electorate chose the sincere Condorcet winner. Given the theorems of social-choice theory concerning the general instability of majority rule, the selection of a Condorcet winner 
with such a high level of confidence is about the best one can hope for in a voting method. This restores some degree of confidence that voting can indeed reflect a majority will among a given set of candidates.

It remains true that a cycle occasionally will occur. In these cases it is more likely that the cycle is an indication of a manipulated outcome than of a cycle in the sincere preferences. One can respond in several ways to such a cycle. We could accept the Kemeny winner as is, even though its legitimacy seems shaky. We could rerun the election, hoping perhaps that A's supporters may respond in kind (which lowers the probability of a cycle), therefore increasing the likelihood of an apparently legitimate outcome. Or, we could adapt the Kemeny rule to say that in the case of a cycle, the alternative that defeats the Kemeny winner is selected, on the grounds that the outcome has most likely been manipulated. If A is sincerely preferred to B, then B's supporters cannot change this. They must hope to engineer a $C$ beats $A$ beats $B$ beats $C$ cycle. In this case, if $B$ is the Kemeny-system winner (that is, the manipulation works), selecting $A$ (which still defeats $B$ in a pairwise comparison) would 'undo' the manipulation. ${ }^{12}$

In sum, the Kemeny function goes a considerable way toward addressing the problems that concern Riker. It selects Condorcet winners, and in the great majority of cases it reassures us about the legitimacy of the choice, because of the absence of a cycle. Even if a cycle is found-to exist, several options remain open. In addition, the Condorcet property means that the Kemeny function is much less vulnerable than are the other two methods to manipulation via variation in the set of candidates. This provides an important defense against another potential threat to the legitimacy of its outcomes.

These properties of the Kemeny function lead me to disagree with Professor Riker's pessimistic view of the theory of democracy. We can indeed find a voting method that selects Condorcet winners and which, while not invulnerable to manipulation, can give a highly reliable signal concerning the outcome's sincerity. Although these properties are not all that one would ideally ask of a voting system, they are also not so little as to convince me to lower my aspirations for voting to the minimal level of Riker's liberal interpretation.

\section{NOTES}

1. See Enelow and Hinich (1983) for a recent clarification concerning these conditions.

2. I ignore the possibility of manipulating the slate of candidates to manipulate the outcome.

3. Here I consider only voting cycles with intransitive strict preferences, not indifference. Some experimentation with the use of cycles containing indifference as a signal show no significant differences in results.

4. For instance, the probability of a cycle in a four-candidate, large electorate election is ap- 
proximately .16 if a large number of voters are selected from an impartial culture. Chamberlin and Cohen (1978) report a much smaller frequency of cycles (4 out of 900) in elections based on a spatial model. Recently, Merrill (1984) checks the incidence of Condorcet winners in several thousand spatial elections and finds them to exist more than 98 percent of the time.

5. This assumption seems quite plausible for plurality voting, in which polling data commonly suffice. If the other systems were in use, it seems likely that polls would obtain rankordered preferences, permitting the necessary forecasts. I ignore the possibility of manipulating polling outcomes by misrepresenting preferences to pollsters.

6. This strategy for manipulating plurality voting is optimal. The strategy for the Borda rule occasionally backfire, defeating $A$ but also $B$, since the strategy may aid $C$ and/or D as well. Although strategies exist that avoid this problem, they involve degrees of information and communication that exceed reasonable bounds for mass elections. It is also possible that strategies exist that manipulate the Borda rule with less chance of creating a cycle, but they would be difficult to ascertain and once again would require nearly perfect information about voters' sincere preferences. We know little about manipulating the Kemeny system, but as with the Borda rule, the strategy noted here is likely to suffice in many cases, if any will.

7. Even under plurality voting, the analysis assumes that rank-ordered preferences are obtained. Although unreal, the assumption permits a comparable analysis of all three voting methods, as well as a demonstration that the existence of a cycle is not always conclusive evidence about manipulability. The strategy for manipulating plurality voting is less likely to generate cycles, and the existence of a cycle is therefore a much weaker signal of manipulation.

8. I selected these cases from among several dozens studied. The cases included here contain all of the interesting findings.

9. Chamberlin and Cohen (1978) generate 300 elections at each of three levels of candidate dispersion relative to voter dispersion in the issue space. I have combined these three sets of elections here. See Chamberlin and Cohen (1978) for further details of the model, including the covariance structure.

10. I generate these elections by adding 1000 newly generated impartial-culture voters to each of the spatial elections, and then dividing each frequency by two and rounding off. The ' $50-50$ ' elections are thus not independent of the spatial elections, but both sets are independent of the impartial-culture elections that are studied.

11. My own hunch is that analyses based on impartial-culture assumptions greatly overstate the likelihood of cycles in naturally occurring elections with many voters. I think it much more likely that the ' $50-50$ ' elections analyzed here are a reasonable best guess about the form of naturally occurring elections.

12. As one referee correctly noted, such a move changes the nature of the voting system. Voters would then have incentives to generate a cycle in which their preferred candidate was the one who defeated the apparent Kemeny-system winner. On both theoretical and practical grounds, this proposal has considerable problems, and it is included here simply to indicate a way in which one might try to foil attempts at manipulation.

\section{REFERENCES}

Arrow, K. (1963). Social choice and individual values. New York: Wiley.

Black, D., and Newing, R.A. (1951). Committee decisions with complementary valuation. Edinburgh: William Hodge.

Chamberlin, J.R., and Cohen, M.D. (1978). Toward applicable social-choice theory: A com- 
parison of social-choice functions under spatial model assumptions. American Political Science Review 72 (December): 1341-1356.

Enelow J., and Hinich, M. (1983). On Plott's pairwise symmetry condition for majority rule equilibrium. Public Choice 40: 317-321.

Farquharson, R. (1969). Theory of voting. New Haven: Yale University Press.

Gibbard, A. (1973). Manipulation of voting schemes: A general result. Econometrica 41 (July): $587-601$.

Kemeny, J. (1959). Mathematics without numbers. Daedulus 88 (Fall): 577-591.

Levenglick, A. (1975). Fair and reasonable election systems. Behavioral Science 20 (January): $34-46$.

McKelvey, R. (1976). Intransitivities in multidimensional voting models and some implications for agenda control. Journal of Economic Theory 12 (June): 472-482.

Merrill, S., III. (1984). A comparison of efficiency of multicandidate electoral systems. American Journal of Political Science 28 (February): 23-48.

Miller, N. (1983). Pluralism and social-choice. American Political Science Review 77 (September): 734-747.

Plott, C. (1967). A notion of equilibrium and its possibility under majority rule. American Economic Review 57 (September): 787-806.

Riker, W. (1978). A confrontation between the theory of social choice and the theory of democracy. Presented at the Annual Meeting of the American Political Science Association.

Riker, W. (1982). Liberalism against populism: A confrontation between the theory of democracy and the theory of social-choice. San Francisco: W.H. Freeman.

Satterthwaite, M. (1975). Strategy proofness and Arrow's conditions. Journal of Economic Theory 10 (October): 187-217.

Schofield, N. (1978). Instability of simple dynamic games. Review of Economic Students 45 (October): 575-594.

Young, H.P. (1974). An Axiomatization of Borda's rule. Journal of Economic Theory 9 (September): $43-52$. 